\title{
ON THE NATURE OF THE EMISSION FROM THE GALAXY NGG 4486
}

\author{
I. S. SHKLOVSKY \\ Sternberg Astronomical Institute, Moscow, U.S.S.R.
}

As is known, a striking peculiarity of the radio galaxy NGC 4486 is the presence of a small and very bright 'jet' in its central part. As it seems to us, the key to the understanding of the nature of the radio galaxy NGG 4486 is the purely continuous spectrum of the above jet, where not even a slightest trace or emission or absorption lines is present.

In the case of the Crab nebula we also meet intense continuous optical emission of some specific nature accompanied by powerful radio emission. We gave some time ago a new interpretation of the continuous optical emission from the Crab nebula. This emission is caused by the same mechanism of non-thermal character, which causes radio emission, namely by relativistic electrons in magnetic fields.

There are reasons to suggest that the nature of the continuous optical emission of the 'jet' of NGC 4486 is similar. From our calculations [1] it follows, that the intensity of the magnetic field in the region of the 'jet' is of the order of $10^{-4}$ gauss, namely approximately the same as in the 'amorphous' part of the Crab nebula. It follows as a result that the energy of the relativistic electrons, responsible for the optical emission of the 'jet' must be of the order of ${ }^{10^{11}}-\mathrm{IO}^{12} \mathrm{eV}$, and their concentration about $5 \times 10^{-9} \mathrm{~cm}^{-3}$. A much larger amount of electrons with energies equalling ${ }^{1} 0^{9}-10^{10} \mathrm{eV}$ should be present in this case. The region of the 'jet' is, consequently, a powerful generator of relativistic particles. Unusual conditions prevailing there, are apparently favourable for the acceleration of particles. Obviously, the acceleration is caused by a Fermi statistical mechanism.

Relativistic electrons formed in the central part of NGG 4486 will diffuse into the surrounding space and in the course of $I-2$ millions of years (the time during which the 'jet' is getting formed) they are filling a considerable part of the volume of NGG 4486 . Wandering through faint interstellar magnetic fields of that galaxy, the relativistic electrons will 
radiate in the range of radio waves, which is the cause of an anomalously high radio emission capacity of NGC 4486 .

From the quantitative theory developed by us it follows that the mean concentration of relativistic electrons with energies $E$ greater than $5 \times 10^{-8} \mathrm{eV}$ is in NGC 4486 of the order of $5 \times 10^{-8} \mathrm{~cm}^{-3}$, and their differential energy spectrum $d n(E)=K / E^{3}$. The total energy of the relativistic electrons is about $5 \times 10^{56}$ ergs over the whole radio galaxy NGC 4486 . The number of particularly energetic 'luminous' relativistic electrons with $E$ about ${ }^{10}{ }^{11-}-\mathrm{IO}^{12} \mathrm{eV}$, located in the region of the 'jet', is millions of times less than the number of less energetic relativistic electrons filling a considerable part of the volume of NGG 4486 and causing its radio emission.

Supposing that the optical emission of the 'jet' continues to exist for $10^{6}$ years with the observed intensity, we shall find that during this interval of time the radiated relativistic electrons loose about $2 \times 10^{55}$ ergs of their energy. This new estimation of the process, which is the initial cause of the anomalous phenomena going on in NGC 4486 , is one and a half order lower than the above estimate (total energy of relativistic electrons in NGG 4486). It may be considered as the lower boundary of the energy emitted for the formation of the 'jet'.

Let us point out that the radio emission of NGC 4486 for $10^{6}$ years, integrated over the spectrum, will be about $10^{54} \mathrm{ergs}$, i.e. considerably less than the amount of energy lost by the electrons of the 'jet' in the course of the same interval of time.

This is explained by the fact that the relativistic electrons in the interstellar medium of NGC 4486 are loosing their energy extremely slowly. They will cause radio emission for at least $5 \times 10^{8}$ years. The 'jet' will get dispersed in the course of that time and its visible traces will disappear.

An extremely interesting and important conclusion follows from this suggestion: there may be observed radio galaxies with absolute and respective radio emission similar to NGG 4486 , but without any peculiarities in their optical rays!

It may be considered that all relativistic electrons that fill the volume of NGG 4486 were found in result of single outbursts. It is most natural, however, to suggest that the 'jets' in NGC 4486 are a recurrent phenomenon and that relativistic electrons that have filled NGG 4486 were formed as a result of 10-20 outbursts.

What is the nature of ' $a$ jet' (or of 'jets')? Two hypotheses may be suggested.

(a) The 'jet' has originated as the result of a certain enormous explosion $5^{18}$ 
in the central part of NGC 4486 . However, it should be accepted in this case that the energy emitted during such an explosion is extraordinarily enormous-it is hundreds of millions times greater than during an outburst of a super-nova. Similar phenomena are unknown in modern physics or astrophysics. Difficulties that arise when such a hypothesis is subsequently developed are extremely numerous.

(b) The anomalous conditions in the central part of NGG 4486 are caused by collisions of massive aggregates (of the type of large globular clusters) containing interstellar gas. These aggregates, extremely numerous in the spheroidal galaxy NGC 4486 , must have velocities of motion about $500-800 \mathrm{~km} / \mathrm{sec}$ and 'frontal' collisions between them may, similarly as in the case of the colliding galaxy Cygnus A, be the cause of a generation of considerable amounts of relativistic particles.

\section{REFERENCE}

[1] Shklovsky, I. S. A.J. U.S.S.R. 33, no. 3, 1955. 